\title{
PREVALENCIA DE OBESIDAD EN ESCOLARES EN DOS COMUNIDADES RURALES
}

\section{Prevalence of obesity in school children in two rural communities}

\author{
${ }^{*}$ Carlos Amílcar Fuentes, *Juan José Guerra, **Cinea Mabeth Abujieres, ${ }^{* *}$ Coster José Coca, \\ **Danilo Fernando Alvarenga, **José Edgardo Rodríguez, ** Mario Román Murcia.
}

\section{RESUMEN}

El continuo incremento de la obesidad en niños constituye un importante problema de salud que requiere de la identificación precisa de su magnitud y tendencias. Objetivo: Establecer la prevalencia de obesidad en escolares del Centro Básico Ingeniero Luis Bográn (CBILB) y el Centro de Educación Básica Marco Aurelio Pinto (CEBMAP) en el mes de agosto del año 2015. Pacientes y métodos: Se realizó un estudio descriptivo, de corte transversal, durante el mes de agosto del año 2015, en escolares de dos Centros Básicos simultáneamente, el Centro Básico Ingeniero Luis Bográn, ubicado en la comunidad de Gualjoco, Santa Bárbara y el Centro de Educación Básica Marco Aurelio Pinto, ubicado en la comunidad de El Coowle, San Manuel, Cortés. Mediante muestreo probabilístico aleatorio estratificado se obtuvo 154 y 93 escolares respectivamente, los cuales fueron evaluados antropométricamente. Los datos se analizaron mediante PASSW Statistics 18. Resultados: Se encontró que la prevalencia de obesidad en CBILB fue de $12.3 \%$, siendo más frecuente en mujeres $(7.14 \%)$ y en edades entre 9-11 años $(7.79 \%)$ y en el CEBMAP fue de $(6 \%)$, siendo más frecuente en hombres $(7 \%)$. Conclusiones: La prevalencia de obesidad en escolares es significativa y varía según sexo y edad.

\section{PALABRAS CLAVE}

Desarrollo infantil, Obesidad, Prevalencia.

\section{SUMMARY}

The continuous increase of obesity in children is a major health problem that requires accurate identification of the magnitude and trends. Objective: Establish the prevalence of obesity in school Basic Engineer Luis Bográn Center

*Doctor en medicina y cirugía. Docente de la Escuela Universitaria de las Ciencias de la Salud. EUCS, UNAH-VS.

** Estudiantes de Medicina, EUCS, UNAH-VS.

Dirigir correspondencia a: abujieres92@yahoo.com

Recibido: 23 de septiembre 2015, Aprobado: 24 de mayo 2015
(CBILB) and the Center for Basic Education Marco Aurelio Pinto (CEBMAP) in August 2015. Patients and methods: A descriptive study of cross section, during the month of August 2015, two schools simultaneously Basic Centers, Basic Engineer Luis Bográn Center, located in the community of Gualjoco, Santa Barbara and the Center for Basic Education Marco Aurelio Pinto, located in the community $\mathrm{El}$ Coowle, San Manuel, Cortés. Using stratified random probability sampling was obtained a sample of 154 and 93 children respectively, which were evaluated anthropometricly. The data were analyzed using Statistics PASSW 18. Results: It was established that the prevalence of obesity in school age children in the Basic Engineer Luis Bográn Center was $12.3 \%$, being more frequent in women $(7.14 \%)$ and ages between 9-11 years $(7.79 \%)$ and in the Center for Basic Education Marco Aurelio Pinto was (6\%), being more common in men (7.3\%). Conclusions: The prevalence of obesity in school age children is significant and varies with sex and age.

\section{KEYWORDS}

Child development, Obesity, Prevalence.

\section{INTRODUCCIÓN}

La Organización Mundial de la Salud (OMS) define la obesidad como una acumulación anormal o excesiva de grasa que puede ser perjudicial para la salud. ${ }^{(1)}$ En la última década la obesidad en escolares ha aumentado a un ritmo alarmante por lo que se ha convertido en un problema de salud pública, aún en países tercer mundistas afectados por la desnutrición. . $^{(2,3)}$

La infancia es la época de la vida en la que se establecen patrones, hábitos y estilos de vida que condicionarán el comportamiento alimentario en la etapa adulta, la adquisición y mantenimiento de obesidad. La obesidad infantil incrementa el riesgo de aparición de patologías cróni- 
cas en la edad adulta, como hipertensión, diabetes mellitus, hiperlipoproteinemia y enfermedades cardiovasculares, así como, disminución en la liberación de hormona del crecimiento, ${ }^{(4)}$ alteraciones respiratorias y problemas ortopédicos en la infancia. Adicionalmente, estos pacientes presentan alteraciones psicológicas y sociales, a menudo relacionadas con la disminución en la autoestima y en la autoconfianza producida por el aislamiento y la discriminación, que condicionarán una menor esperanza de vida y un aumento en el deterioro de la calidad de vida. ${ }^{(5,6)}$

No existen criterios estandarizados para definir obesidad infantil, ni que permitan evaluar el riesgo ni los resultados en estas edades, lo que dificulta el conocimiento exacto de su magnitud. ${ }^{(7,8)}$ Actualmente la estimación de la obesidad en la infancia tanto a nivel clínico como epidemiológico, se realiza a partir del índice de masa corporal (IMC), definiendo la obesidad mediante la utilización de percentiles. ${ }^{(9)}$

El sobrepeso y la obesidad han aumentado en los últimos 30 años al grado de convertirse en un problema de proporciones epidémicas en adultos y en niños, tanto en países desarrollados como en aquéllos en vías de desarrollo. Hasta hace poco, prácticamente no se hablaba de obesidad en la infancia y en la adolescencia, y la desnutrición dominaba los espacios en revistas especializadas de nutrición y salud ${ }^{(10)}$ por la infinidad de factores asociados a la obesidad en la edad pediátrica entre los cuales tenemos: una dieta rica en grasas, más de 4 horas diarias de televisión, familia monoparental, hijo único o adoptado, el menor de los hermanos, madre añosa, menor peso al nacer y consumo de bebidas con alto contenido de azúcares. ${ }^{(11,12)}$

La OMS estima que por lo menos 300 millones de personas en el mundo son obesos y unos 700 millones sufren de sobrepeso; las proyecciones estiman que para 2015 al menos 20 millones de niños menores de 5 años tendrán sobrepeso ${ }^{(13)}$ y alrededor del $14 \%$ son obesos al comenzar la escuela. ${ }^{(10)}$ Un niño obeso tiene $12.6 \%$ más probabilidades de tener diabetes mellitus y $9 \%$ más de ser hipertenso a edad temprana que niños no obesos. ${ }^{(14)}$
Entre 1990-1999 y 2000-2006 para otros países latinoamericanos, se informa un aumento de la prevalencia: El Salvador de 3.9\% a 5.8\%, República Dominicana de 6.9 a $8.6 \%$, Honduras de $2.4 \%$ a $5.8 \%$, Nicaragua de $3.5 \%$ a $7.1 \%$ y Perú de 9.9 a $11.8 \% .^{(9)}$

En Honduras hay tres estudios publicados sobre obesidad, el primero donde se evalúa tratamiento quirúrgico en cinco adultos en 1974. El segundo, del año 2000, que estudió la población escolar de Tegucigalpa y reportó, acorde al índice de masa (IMC), que el $2 \%$ de éstos niños tenían un bajo peso, $13 \%$ sobrepeso y $6 \%$ obesidad, además se identificó una asociación entre obesidad y la presencia de un pariente cercano con diabetes mellitus tipo 2 (DMT2); se encontró que si se tiene un familiar con DMT2 es aproximadamente tres veces más probable encontrar un niño obeso. ${ }^{(15)}$ El tercero, del año 2010, estudió las conductas alimentarias y actividad física en escolares de Tegucigalpa el cual arrojó que el $65.4 \%$ de los escolares consumían en su vivienda comida saludable, $48.6 \%$ de las meriendas eran altas en grasas y $15.8 \%$ altas en carbohidratos; con respecto a la actividad física $53.2 \%$ realizaban actividad física intensa y únicamente el $27 \%$ realizaba actividad física en la escuela. ${ }^{(16)}$

Por lo tanto, el objetivo de la investigación fue establecer la prevalencia de obesidad en los escolares del Centro Básico Ingeniero Luis Bográn (CBILB) y el Centro de Educación Básica Marco Aurelio Pinto (CEBMAP) en el mes de agosto del año 2015.

\section{PACIENTES Y MÉTODOS}

Se realizó un estudio descriptivo, de corte transversal, durante el mes de agosto del año 2015, en escolares de dos Centros Básicos simultáneamente, el Centro Básico Ingeniero Luis Bográn (CBILB), ubicado en la comunidad de Gualjoco, Santa Bárbara y el Centro de Educación Básica Marco Aurelio Pinto (CEBAP), ubicado en la comunidad de El Coowle, San Manuel, Cortés.

Los criterios de inclusión fueron: estudiante matriculado en el Centro Básico Ingeniero Luis Bográn y en Centro de Educación Básica Marco Aurelio Pinto, estudiante en edad comprendida entre 6 y 14 años para CBILB y entre 6 y 12 años 
para CEBMAP y con autorización firmada por sus padres para participar en la investigación.

La población estuvo constituida de la siguiente manera: en el CBILB, eran 325 escolares (167 niños, 158 niñas) y en el CEBMAP 198 (117 niños y 81 niñas).

La muestra fue calculada mediante muestreo probabilístico aleatorio con fórmula para muestras finitas, estratificandolo según sexo, obteniendo: CBILB 154 escolares (80 niños y 74 niñas) y en el CEBMAP 93 (55 niños y 38 niñas).

Los datos etarios se obtuvieron mediante el registro de matrícula y fecha de nacimiento brindada por el centro educativo. Los escolares fueron pesados en posición de pie, con su uniforme puesto, en una balanza previamente calibrada. La talla se midió, estando el niño descalzo y en posición de pie, utilizando el tallímetro que viene adyacente a la balanza en centímetros. Ambas mediciones se realizaron por un mismo investigador. El índice de Masa Corporal (IMC) se calculó a partir de las medidas de peso y talla $(\mathrm{IMC}=$ peso $[\mathrm{kg}] /$ talla $[\mathrm{m} 2])$ y se utilizó para clasificar a la población según su estado ponderal estableciéndose los puntos de corte según la European Childhood Obesity Group (ECOG); bajo peso <p25, normopeso, $>$ p25 y <p85, sobrepeso >p85 y obesidad >p95. Los datos se analizaron mediante PASSW Statistics 18, utilizando frecuencias, tablas de contingencia y gráficos descriptivos.

Para fines del estudio los datos recolectados en ambos centros básicos se unieron, teniendo así una muestra más representativa.

\section{RESULTADOS}

En el Centro Básico Ingeniero Luis Bográn, de los 154 escolares evaluados, 57 (37\%) se encontraban entre 6-8 años, 66 (42.9\%) 9-11 años y $31(20.1 \%)$ 12-14 años de edad. 80 $(51.95 \%)$ eran hombres y $74(48.05 \%)$ mujeres.

Del total $104(67.5 \%)$ se encontraban por debajo del percentil $85(P<85), 31(20.1 \%)$ entre los percentiles 85 y $95(P>85$ y $P<95)$ y $19(12.3 \%)$ por encima del percentil $95(\mathrm{P}>95)$, por lo tanto la prevalencia de obesidad en escolares fue de $12.3 \%$. Según el sexo, la prevalencia de obesidad en mujeres fue de $10(13.51 \%)$ (Ver tabla No.1) y según la edad la prevalencia de obesidad en escolares entre 9-11 año fue de 12 (7.79\%). Ver gráfico No.1.

Tabla No.1: Prevalencia de obesidad en escolares en CBILB según sexo

\begin{tabular}{|c|c|c|}
\hline Percentiles (p) & Hombre & Mujer \\
\hline P< 85 & $58(72.5 \%)$ & $46(62.16 \%)$ \\
\hline P85 y P95 & $13(16.25 \%)$ & $18(24.33 \%)$ \\
\hline$>$ P95 & $9(11.25 \%)$ & $10(13.51 \%)$ \\
\hline TOTAL & $80(100 \%)$ & $74(100 \%)$ \\
\hline
\end{tabular}

Fuente: Instrumento obesidad en escolares

Gráfico No. 1: Prevalencia de obesidad en escolares de CBLIB según edad

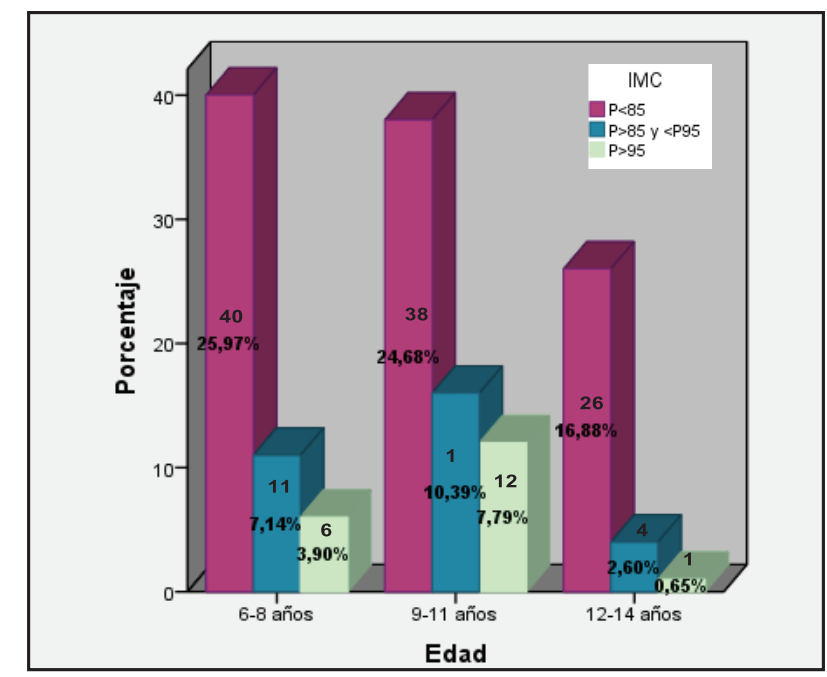

Fuente: Instrumento obesidad en escolares

Tabla No. 2: Distribución del estado nutricional de escolares en CBMAP

\begin{tabular}{|c|c|c|}
\hline Percentiles $(\mathbf{p})$ & Hombre & Mujer \\
\hline P<85 & $41(74.5 \%)$ & $30(78.9 \%)$ \\
\hline P85 y P95 & $10(18.2 \%)$ & $6(15.8 \%)$ \\
\hline P $>95$ & $4(7.3 \%)$ & $2(5.3 \%)$ \\
\hline TOTAL & $55(100 \%)$ & $38(100 \%)$ \\
\hline
\end{tabular}

Fuente: Instrumento obesidad en escolares 
En el Centro de Educación Básica Marco Aurelio Pinto de los 93 escolares evaluados $6(6.5 \%)$ se encontraban por encima del percentil 95 ( $P>95)$, lo que constituye la prevalencia de obesidad en dicho centro, $16(17.2 \%)$ están por encima del percentil $85(\mathrm{P}>85)$ y $71(76.3 \%)$ por debajo del percentil $85(P<85)$. Según el sexo la prevalencia en varones fue de 4 (7.3\%) Ver tabla No. 2.

\section{DISCUSIÓN}

La obesidad en niños estará determinada por aspectos genéticos y culturales. Según la Encuesta de Demografía y Salud ENDESA 2012, del Instituto Nacional de Estadística de la República de Honduras, entre los aspectos culturales que favorecen la obesidad en niños, se incluyen cambios en los patrones alimenticios tradicionales por importados, consumo excesivo de bebidas carbonatadas, escasa participación de los padres en la preparación de alimentos aduciendo a esto razones de trabajo, priorización en cuanto a alimentación al hijo mayor, etc. $^{(17)}$ Hechos que se practican con mayor intensidad día con día y que favorecen al incremento de la prevalencia de obesidad en niños.

En un estudio realizado en escuelas públicas de Tegucigalpa, Honduras en el año 2000, la prevalencia de obesidad fue de $6.4 \%,{ }^{(16)}$ lo que difiere significativamente con el $12.3 \%$ encontrado en este estudio en lo correspondiente al Centro Básico Ingeniero Luis Bográn, sin embargo no varía en gran medida con respecto al $6 \%$ encontrado en el Centro de Educación Básica Marco Aurelio Pinto. En comparación con otros países los datos encontrados son variables; en Argentina en el 2005 se estimó que la prevalencia de obesidad en escolares fue de 13.8\%, en México en el 2011 se estimó en $23 \%$ y en España en el 2013 en $23.5 \%$. $^{(9,16,18)}$
En escolares de Tegucigalpa se encontró mayor prevalencia de obesidad en niñas en edades ntre 11-14 años. El 1.9\%; en este estudio también fue mayor en las niñas del CBILB con $7.14 \%$, sin embargo la edad en la que más se presentó fue entre los 9-11 años con 7.79\%, contrario al hallazgo en el CEBMAP en donde la prevalencia fue mayor en los varones $7 \% .{ }^{(15)}$

En cuanto al sexo en que más prevalece, según este estudio en el CBILB, es en niñas con $7.14 \%$ en contraste con países como México en donde el sexo con más obesidad son los niños con $29 \%$ y Argentina en donde también son los niños con un $14.8 \%$, mostrando una diferencia significativa en relación al 5.19\% encontrado en niños en el presente estudio para el CBILB y el $7.3 \%$ para el CEBMAP. ${ }^{(9,16,19)}$

En conclusión la prevalencia de obesidad en escolares en el Centro Básico Ingeniero Luis Bográn es más frecuente en mujeres y en edades entre los 9 y 11 años. En el Centro Básico Marco Aurelio Pinto es más frecuente en hombres. Es importante hacer notar que los datos finales del estudio, avalan el hecho de que la obesidad en niños representa un importante problema de salud, y que están en concordancias con las estadísticas internacionales.

Se recomienda realizar campañas educativas sobre las consecuencias a corto y largo plazo que provoca la obesidad y el consumo de comidas rápidas y bebidas carbonatadas. Brindar talleres participativos de nutrición a padres de familia y maestros para de esta manera incentivar las meriendas saludables y concientizar sobre la importancia de realizar actividad física mínimo tres veces por semana.

\section{BIBLIOGRAFÍA}

1. Basain Valdés JM, Valdés Alonso MC, Miyar Piegia E, Peréz Martínez M, Tase Pelegrin S, Ramos Arteaga ME. Malnutrición por exceso e hipertensión arterial en niños y adolescentes en un área de salud. Revista Archivos Médico de Camagüey. 2015; 19(3): 220-228. Noviembre-Diciembre.

2. Piero A, Rodríguez E, Gonzales L, López A.
Sobrepeso y obesidad en un grupo de escolares españoles. Revista Chilena de Nutrición. 2014;41 (3): 264- 271. Septiembre.

3. Hernández Álvarez G. Prevalencia de sobrepeso y obesidad, y factores de riesgo, en niños de 7-12 años, en una escuela pública de Cartagena septiembre-octubre de 2010. (Tesis para optar el título de Magíster 
en Salud Pública). Cartagena. Universidad Nacional de Colombia.2011.

4. Berta EE, Fugas VA, Walz F, Martinelli MI. Estado nutricional de escolares y su relación con el hábito y calidad del desayuno. Revista Chilena de Nutrición. 2015;42(1): 45-52. Marzo.

5. Raimmant $X$, Verdugo F. Actividad física en la prevención y tratamiento de la obesidad infantil. Revista Médica Clínica Las Condes. 2011; 23(3): 218-225. Septiembre.

6. Briz Hidalgo FJ, Cos Blanco Al, Amate Garrido AM. Prevalencia de obesidad infantil en CEUTA. Estudio PONCE. Revista Nutrición Hospitalaria. 2010; 22(4): 471477. Octubre-Diciembre.

7. Atalah E, Urteaga C, Rebolledo A, Delfín S, Ramos R. Prevalencia de obesidad en escolares de la región de Aysén. Revista Archivos de Pediatría del Uruguay. 2011; 72 (3): 235-241.Marzo.

8. Araujo Herrera O. Síndrome metabólico en la infancia, un enfoque para la atención primaria. Revista Cubana de Pediatría. 2015; 87(1):82-91.Enero-Marzo.

9. Villanueva Montemayor D, Hernández Herrera RJ, Salinas Martínez AM, Mathiew Quiros $A$, Sánchez Espinoza M. Prevalencia de obesidad infantil en niños entre 6 y 14 años de edad en una Unidad de Medicina Familiar del IMSS. Revista Pediatría de México. 2011; 13(4):151-154. Octubre- Diciembre.

10. Kaufer-Horwitz M, Toussaint G. Indicadores antropométricos para evaluar sobrepeso y obesidad en pediatría. Boletín Médico del Hospital Infantil de México. 2008; 65(6): 502-518.

11. Girón K, Espinoza L. Epidemiología de la Obesidad en la Consulta Externa de Endocrinología del Departamento de Pediatría. Hospital de Especialidades del Instituto Hondureño de Seguridad Social (IHSS). Período
2002-2004.Revista Médica de los Post Grados de Medicina UNAH 2006; 9(2):243248.

12. Dodd $A H$, Briefel $R$, Cabili $C$, Wilson $A$, Crepinsek MK Disparities in consumption of sugar-sweetened and other beverages by race/ethnicity and obesity status among United States schoolchildren Journal of Nutrition Education and Behavior. 2013; 45(3): 240-249.

13. Quintana-Guzmán EM, Salas-Chaves MP, Cartín-Brenes M. Índice de masa corporal y composición corporal con deuterio en niños costarricenses. Acta Pediátrica de México. 2014; 35:179- 189.

14. Lizardo AE, Díaz, A. Sobrepeso y obesidad infantil. Revista Médica Hondureña 2011; 79(4):208-13.

15. Rivera MF. Obesidad en condiciones de pobreza. Estudio epidemiológico en escolares de escuelas públicas de Tegucigalpa, Honduras, 2000. Revista Médica Hondureña. 2005; 73(1):10-14. Enero-Febrero-Marzo.

16. Rivera MF, Rivera IC. Conductas alimentarias y actividad física en niños escolares de Tegucigalpa. Revista Médica Hondureña. 2010; 78(2):65-69.

17. Encuesta de Demografía y Salud ENDESA 2012. Instituto Nacional de Estadística. Tegucigalpa M.D.C Mayo. 2013.

18. Padilla IS. Prevalencia de sobrepeso-obesidad y factores asociados con valor predictivo-preventivo en escolares de 6 a 11 años de Río Gallegos, Santa Cruz, Argentina. Revista Salud Colectiva. 2011; 7(3): 377388. Septiembre-Diciembre.

19. Plaza Almeida J, Siurana Rodríguez JM, Vergara Gonzáles LI, Mateos Rodríguez F, Romero Belluga MI. Prevalencia de obesidad en escolares. Revista Clínica de Medicina de Familia. 2013; 2(3) 106-110. Junio. 